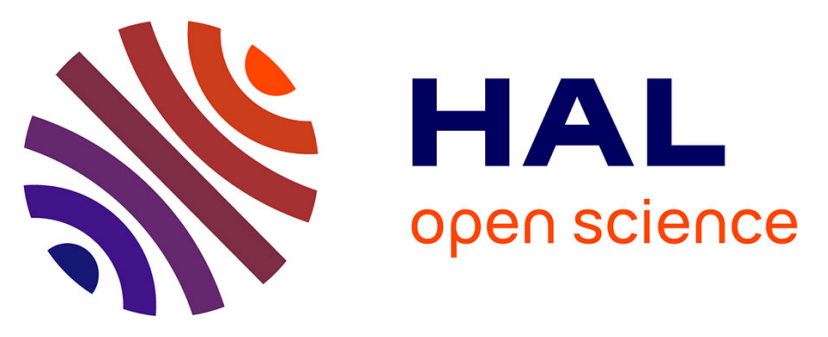

\title{
Seismic Velocity Response to Atmospheric Pressure Using Time-Lapse Passive Seismic Interferometry
}

Chloé Gradon, Florent Brenguier, Johannes Stammeijer, Aurélien Mordret, Kees Hindriks, Xander Campman, Richard Lynch, Pierre Boué, Malgorzata Chmiel

\section{To cite this version:}

Chloé Gradon, Florent Brenguier, Johannes Stammeijer, Aurélien Mordret, Kees Hindriks, et al.. Seismic Velocity Response to Atmospheric Pressure Using Time-Lapse Passive Seismic Interferometry. Bulletin of the Seismological Society of America, 2021, 10.1785/0120210069 . hal-03320516

\section{HAL Id: hal-03320516 https://hal.science/hal-03320516}

Submitted on 16 Aug 2021

HAL is a multi-disciplinary open access archive for the deposit and dissemination of scientific research documents, whether they are published or not. The documents may come from teaching and research institutions in France or abroad, or from public or private research centers.
L'archive ouverte pluridisciplinaire HAL, est destinée au dépôt et à la diffusion de documents scientifiques de niveau recherche, publiés ou non, émanant des établissements d'enseignement et de recherche français ou étrangers, des laboratoires publics ou privés. 
1. Seismic velocity response to atmospheric pressure

2 using time-lapse passive seismic interferometry

3 Chloé Gradon (UGA, Sisprobe), Florent Brenguier (UGA),

Johannes Stammeijer (PDO), Aurélien Mordret (UGA),

Kees Hindriks (Shell), Xander Campman (Shell),

Richard Lynch (Sisprobe), Pierre Boué (UGA)

and Malgorzata Chmiel (ETH)

4

August 12, 2021

5 Corresponding Author: Chloé Gradon (chloe.gradon@univ-grenoble-alpes.fr),

6 Université Grenoble Alpes, ISTerre, CS 40700, 38058 GRENOBLE Cedex 9,

7 France

8

9

10

11

\section{Declaration on competing interest}

The authors acknowledge there are no conflicts of interest recorded.

Abstract
Seismic velocities in the shallow crust down to a few kilometers depth
show a remarkable sensitivity to stress perturbations due to the presence
of compliant pores, cracks, fractures, and faults. Monitoring temporal




\section{${ }_{43} \quad 1$ Introduction}

44 Improving our ability to monitor stress changes occurring at seismogenic depth

45 is crucial to better understand processes associated with the generation of large

46 earthquakes (Kato \& Ben-Zion, 2021). Both laboratory and field experiments

47 have shown that stress perturbations as small as a few kiloPascal have a measur- 
able impact on seismic velocities (e.g. Yamamura et al. (2003)). This stress dependence is known to be mostly controlled by the opening/closing of microcracks due to changes in effective pressure. Continuous seismic velocity monitoring has been successfully applied to study controlled fluid injection, environmental, tectonic and volcanic processes both at a laboratory scale (Scuderi \& Collettini, 2016), and in nature using controlled sources (Niu et al. (2008), Tsuji et al. (2018), Doetsch et al. (2018)) and ambient seismic noise (Sens-Schönfelder \& Wegler (2006), Brenguier et al. (2008a)).

Better understanding velocity changes induced by environmental perturbations is key in unveiling transients associated with deeper tectonic or volcanic processes. These environmental, exogenous processes show a variety of time scales ranging from hours for tidal-induced strain (Yamamura et al., 2003) to days or months for rainfall-induced pore pressure changes (Rivet et al. (2015), Clements \& Denolle (2018)) and atmospheric pressure loading (Silver et al., 2007) with a strong seasonal, yearly component (Wang et al., 2017). In this paper we focus on the effects of atmospheric, barometric pressure temporal changes on near-surface seismic velocities following the seminal paper of Silver et al. (2007). In order to isolate the atmospheric pressure effect we conduct a noise-based, passive seismic monitoring experiment (Sens-Schönfelder \& Wegler (2006), Brenguier et al. (2008a)) in an arid location in the middle of the Oman desert thus strongly reducing the potential effects of rainfall activity or oceanic tidal loading.

We monitor seismic velocities following a workflow similar to Olivier et al. (2015) and measure temporal changes of the travel time of coda-waves retrieved from noise-correlations between strings of geophones located in 5 different wells in the frequency range 10 to $50 \mathrm{~Hz}$. By using four months of continuous seismic records from 56 borehole geophones located down to $365 \mathrm{~m}$ depth, we find a consistent trend of seismic velocity change over the entire study area. While the short-term transients (a few weeks) of velocity changes show a striking 
similarity with atmospheric pressure variations, we do not observe any longterm, seasonal trend as seen in the atmospheric pressure data. We use the model of Roeloffs (1996) to evaluate the effective pressure in the saturated nearsurface using the fully coupled (Biot) equations of elastic deformation and pore fluid flow taking the atmospheric pressure as the source of aquifer loading. By showing a long-term equilibrium between the confining and the pore pressure, this model succeeds in explaining the reduction of seasonal velocity changes and provides a framework for assessing poroelastic parameters such as hydraulic diffusivity. Finally, we use this model to calibrate the observations of seismic velocity variations and find a step-change velocity drop that could be related to production-rate variations at the deeper reservoir.

\section{Data processing}

We use a dataset composed of 4 months of continuous seismic records from 56 , three-component geophones spread over five observation wells located above an oil reservoir in the desert of the Sultanate of Oman (Figure 1, see Data and resources section). The top geological unit is mostly composed of carbonates with an average $\mathrm{P}$-wave velocity of $2625 \mathrm{~m} / \mathrm{s}$ down to about $250 \mathrm{~m}$ depth, followed by alternating carbonate and shale layers and a domed reservoir at an average depth of about $370 \mathrm{~m}$. An aquifer is also present with a water level recorded at around $50 \mathrm{~m}$ below the surface during the experiment.

The geophones are distributed in five seismic monitoring wells (SMW). Four wells are located close to the production area (SMW1, SMW2 SMW3, and SMW4) and one well is about $1 \mathrm{~km}$ away (SMW5). The minimum and maximum distances between two wells are $255 \mathrm{~m}$ and $1775 \mathrm{~m}$, respectively (Figure 1-a). There are eight geophones in each well with an average spacing of $20 \mathrm{~m}$, covering depths ranging from 190 to $330 \mathrm{~m}$ below the surface (Figure 1-b and c). The dataset consists of 122 days (four months) of ambient seismic noise recorded 
from the beginning of August to the end of November 2019. The raw data was recorded at $4 \mathrm{kHz}$ and downsampled at $250 \mathrm{~Hz}$ to reduce computational cost.

Figure 2 shows the averaged daily spectrograms for the $\mathrm{Z}$ component of two sensors in wells SMW3 and SMW5. These spectrograms are obtained by summing daily spectrograms over the 122 days of the study, highlighting the average noise variations over a day. In this example, we show data from the deepest sensors in each well, located at 321 and 362 meters below the surface, respectively. For both sensors, most of the energy is observed below $50 \mathrm{~Hz}$. We explain the difference of amplitudes between both wells by the fact that SMW3 is located above the production area while SMW5 is farther away. The decrease of energy during the nights and lunch breaks indicates that the dominant source of noise is related to human activity around the production area. The emitted noise is strong enough to be clearly measured at the bottom of the wells above the production area. We also see clearly monochromatic components at 50 and $100 \mathrm{~Hz}$ associated with the electrical network.

We first cross-correlate the 122 days of data using 15-minute time windows and a spectral normalization scheme to minimize the impact of the microseismicity present in the area. We only compute correlations over the ZZ, EE and NN components, which are independent, to optimize signal to noise ratio without increasing computational costs. We work between $10 \mathrm{~Hz}$ and $50 \mathrm{~Hz}$ to take advantage of the increased energy in this frequency band. Figure 3-a shows the cross-correlations (CC) according to calendar time on the y-axis for the EE component between the shallowest sensor of SMW3 and the shallowest sensor of SMW4. Each row corresponds to a 15-minute cross-correlation, with colors representing amplitude. The red and black dotted lines are the theoretical arrival for the $\mathrm{P}$ and $\mathrm{S}$-wave in the first layer, respectively. By studying the variation in the cross-correlations over 3 days, we can observe two significantly different waveforms that are correlated with active and quiet periods on the production site. In order to obtain more stable results, we focused on "nighttime" 
cross-correlations corresponding to a period ranging from $20 \mathrm{~h} 00$ to $05 \mathrm{~h} 00$.

We apply the Moving Window CS method (MWCS), also called doublet method, (Poupinet et al. (1984), Brenguier et al. (2008b)) on the negative and positive part of the coda of the correlations to estimate relative travel time variations $(d t / t)$ in the medium. Typically, $d t / t$ time series are obtained by measuring the arrival time difference $(d t)$ between wavepackets in a test trace, i.e. a trace associated to a calendar time T, and a reference trace. In the MWCS method, we measure the $d t$ for a set of time windows in the coda of the traces.

The reference trace used to measure velocity changes is usually the stack of all the good-quality cross-correlations over the study period. This reference must be similar enough to each test trace to allow for time shift measurements. In this study, we use the normalized correlation coefficient between the reference trace and the test traces to measure this similarity. We estimate empirically that a correlation coefficient of 0.8 is necessary to obtain reliable results. Studying the evolution of the cross-correlation over the 122 nights (Figure 3-b), we observe that we have remarkable stability over sub-periods ranging from 3 weeks to 1.5 month. There is also a very clear change in the cross-correlation waveform between each stable sub-period, allowing us to identify them visually (horizontal dotted lines).

Preliminary tests show that using a single reference over the four-month period of the study will not allow us to satisfy the criteria of high coherence between the test and the reference correlations. The clear changes in the correlation traces in Figure 3-b, easily explain why that is the case. Reviewing the correlation coefficient for every sensor pairs, we determined a set of subperiods where the reference and test traces showed sufficient similarity. In Figure 3-b, the references associated to each subperiod are plotted in black, blue and red, with their associated correlation coefficient with test traces obtained by stacking the cross-correlation over three nights represented in Figure 3-c. We chose three-night stacks as a compromise between stability and time resolution. 
We apply the MWCS method over each subperiod using non-overlapping three-night stacks. The coda part of the trace used here starts $0.2 \mathrm{~s}$ after the theoretical arrival time of the S-wave and end at a lapse time of two seconds. We measure the time-shifts in the 10 to $50 \mathrm{~Hz}$ frequency band, and use 0.4 -second time windows that correspond to four periods of the lower frequency signal. To simplify our analysis, we compute the $d t / t$ for each sensor pair over components $\mathrm{ZZ}, \mathrm{EE}$ and NN (thin lines figure 4) and derive one average per well pair (thick

lines). We finally make the assumption that velocity changes are homogeneous over the region sampled by these coda-waves (Brenguier et al. (2008a)) and use the standard relation $d t / t=-d v / v$ to convert relative travel time into relative velocity changes. As the averaged velocity measurements overlap, we are able to reconstruct the total velocity variations by matching the values at the beginning and end of each subperiods, which are represented in Figure 4 by different colors. In practice, these values match without the need for correction in most cases. The gaps in the measurements are due to missing data or lack of high-quality correlations.

To make sure that using several references does not affect a possible long term trend in the $d v / v$ measurements, we used a single reference and 17-night correlations stacks. At this resolution, the similarity between the reference over the four months and test trace was sufficient to obtain stable results. The trend using a single or multiple references is the same, showing that no long term bias was introduced using our method.

\section{Results and interpretation}

For each well we compute an average velocity variation over all pairs of sensors/components and derive one velocity variation per well by averaging all its associated velocity change measurements (Brenguier et al. (2014), SensSchönfelder et al. (2014)). The results are displayed in Figure 5-b using black 
lines. The grey area represents the uncertainties on the measurement obtained by averaging the standard deviations of sensor pair measurements between wells. By considering studies of the spatial sensitivity of coda-wave travel-time change measurements (Snieder et al. (2019)) we evaluate that our observations of Figure 5-b relate to mostly S-wave velocity changes within a region of about $500 \mathrm{~m}$ around the wells at an average sensor depth of about $260 \mathrm{~m}$. The sensitivity of each velocity change time series thus overlap spatially and cover most of the studied area shown on Figure 1. The great similarity of velocity change time series between all wells of Figure 5-b supports the interpretation of a homogeneous velocity change over the entire studied area.

During the studied period, the global production rates over the site are stable. No rainfall was reported and we found that stress perturbations from both tidal oceanic loading and solid earth tides are an order of magnitude smaller than atmospheric pressure variations after smoothing using a three-day averaging moving window. Figure 5-a shows the in situ barometric pressure. It shows peak-to-peak diurnal variations of about $500 \mathrm{~Pa}$, middle-term changes with periodicity from a few days to a few weeks of maximum $1 \mathrm{kPa}$ amplitude and a longterm, seasonal increase of about $2.5 \mathrm{kPa}$. For comparison with rainfall-induced loading, $1 \mathrm{kPa}$ corresponds to the loading from $100 \mathrm{~mm}$ of rainfall which is quite strong meaning that atmospheric pressure loading might be often dominant in the response of the shallow crust to environmental changes.

Interestingly, barometric pressures and seismic velocities show a direct correlation from the beginning of August to mid-September during a strong transient of barometric pressure change lasting about 30 days (minima around August 10 and September 10 and maximum around August 20). The response of a porous medium to loading from atmospheric pressure variations has been described by Van Der Kamp \& Gale (1983). Using the constitutive relations for an isotropic coupled fluid-saturated porous elastic medium from Rice \& Cleary (1976), Van Der Kamp \& Gale (1983) showed that under undrained conditions, meaning 
without considering fluid flow and pore pressure migration, pore pressure $(P o)$ is directly proportional to the confining pressure $(P c)$ induced by barometric pressure loading. The coefficient of proportionality, ranging from 0 to 1 , is also called the Skempton's coefficient $\alpha$ with a value of $0.5(P o=\alpha P c)$. This value is low compared to what is found in laboratory measurements (Ahmadinejad \& Kivi, 2021), but is still realistic in an in-situ context (Roeloffs, 1996). Doetsch et al. (2018) observed that seismic velocity changes $(d v / v)$ were proportional to effective pressure $P e=P c-P o$, where $P o$ is pore pressure. Utilizing Skempton's coefficient leads to $d v / v \propto P e=P c(1-\alpha)$.

This direct proportionality between velocity changes and the confining, atmospheric pressure can explain the early velocity transient, but long-term velocity changes do not show the increasing trend this theory would predict considering the rise of atmospheric pressure from August to December. The lack of long-term velocity increase suggests a process leading to an increase of pore pressure associated with a decrease of velocity. This situation is typically reached under drained conditions involving pore pressure migration (Roeloffs, 1996). Following the same constitutive laws as Van Der Kamp \& Gale (1983), Roeloffs (1996) studies the effect of pore pressure variations induced by atmospheric pressure changes in the case of a vertical, downward migration of fluids from the top of the water table that is directly connected to the atmosphere. In this case, pneumatic diffusion (diffusion of the air in the pores in the unsaturated zone)is neglected (see sketch on Figure 6).

Equation 37 of Roeloffs (1996) describes a harmonic solution for pore pressure according to the frequency of atmospheric pressure changes $(\omega)$, the depth from the top of the water table $(\mathrm{z})$ and the hydraulic diffusivity (c):

$$
\operatorname{Po}(z, \omega, c) / P c(\omega)=\alpha+(1-\alpha) \cdot \exp [-\sqrt{i \omega / c} \cdot z]
$$

If $\alpha$ is significantly different from 1 , as is the case here $(\alpha=0.5)$, we see different 
behaviors at short and long periods (or small and large depths). When the depth is large or for short-term transients (large $\omega$ ), this solution reduces to the undrained case discussed earlier where the pore pressure is directly proportional to the confining, atmospheric pressure ( Figure 6-a). For small depth or longterm pressure variations, the second term representing pore pressure migration dominates and eventually tends to the boundary condition at $\mathrm{z}=0$ for which the pore pressure equals the confining pressure thus leading to a 0 net change of seismic velocities (Figure 6-b).

In order to directly compare this model to our observations we derive a synthetic velocity change time series by using the following relation:

$$
d v / v=K \cdot(P c-P o)
$$

where the effective pressure $P c-P o$ is computed as the difference between the atmospheric pressure and the atmospheric pressure-induced pore pressure. $\mathrm{K}$ is the sensitivity of velocity changes to effective pressure perturbations in $\mathrm{Pa}^{-1}$. To get $P o$, we convolve the impulse response of the harmonic solution for pore pressure variation of eq. 1 to the in-situ atmospheric pressure variation time series. We computed this model for a coarse range of depths and diffusivity coefficients. Figure 5-b shows in red the best fit of this model to the observed velocity changes at $50 \mathrm{~m}$ below the water table level ( $\mathrm{z}=100 \mathrm{~m}$ below the surface). This is above the first sensors in the wells, and could be due to a higher sensitivity of our method to velocity changes at shallower depth. Indeed, the scattering is expected to be stronger close to the surface, resulting in better sampling of the medium by the coda waves used in our measurements. The associated value for the diffusivity is of the order of $100 \mathrm{~m}^{2} / \mathrm{s}$, which is consistent with values found in the literature for carbonates (Roeloffs, 1996). The estimated value for the sensitivity of velocity changes to effective pressure changes over the first month were the correlation between our model and the measurement is the best is $K=6.3 \cdot 10^{-7} \mathrm{~Pa}^{-1}$. This value is typically in the range of values previously 
observed in-situ for environmental changes,with sensitivity ranging from $10^{-9}$ to $10^{-6}$ measured by different teams (Yamamura et al., 2003).

It is important to note that our model is not well constrained. Figure 7 shows different models associated with 9 combinations of depths $(\mathrm{z}=[100,150$, $250] \mathrm{m})$ and diffusivities $\left(\mathrm{c}=[10,100,1000] \mathrm{m}^{2} / \mathrm{s}\right)$. While most models still display strong long-term trends, we see that model 6 is very similar to the one showed Figure 5, and that model 3 shows a better fit. We discarded those models due to the high value of $c=1000 \mathrm{~m}^{2} / \mathrm{s}$. However, according to Roeloffs (1996), this number is typical for karsts involving highly diffusive vertical fractures, and further study would be necessary to better constrain the depth and diffusivity in this analysis.

Interestingly, around October 10, the velocity change observations deviate from the modeled effective pressure by showing an additional step change drop of velocity of about $-1 \cdot 5 \cdot 10^{-4}$. Considering the estimated velocity-pressure sensitivity discussed above, we find that this velocity drop corresponds to a reduction of confining pressure or increase of pore pressure of $240 \mathrm{~Pa}$. This value is quite small but the similarity of velocity variations between all wells indicates that it is likely widespread over the studied area. The wells also overlay an oil and gas reservoir. It is possible that the changes in production rates are causing this step-change decrease of velocity by causing a dilatational expansion of the overburden. We only have access to global rates across the site and cannot conclude from the available production data. Moreover, such a weak stress perturbation of $240 \mathrm{~Pa}$ is probably hardly detectable by other means such as GPS or InSAR, nor by micro-seismicity monitoring, as it probably does not affect the integrity of the overburden. 


\section{${ }_{313}$ Acknowledgments}

\section{Data and Resources}

\section{Conclusions}

By measuring time-lapse changes of seismic velocities within an aquifer located in an arid desert using passive coda-wave interferometry, we are able to observe velocity changes as small as $0.01 \%$ over four months with a temporal resolution of three days. The comparison of our results to barometric pressure time-series shows that the aquifer responds as an undrained poro-elastic medium for shortterm barometric pressure variations of a few weeks but that fluid migration plays an important role at a seasonal time scale. The direct correlation between atmospheric loading and velocity variations that we observe is consistent with Silver et al. (2007) active source monitoring experiment in the case of the farfield experiment with wells separated by 30 meters. By allowing the calibration of seismic velocity changes to stress perturbations, we are able to detect a transient effective pressure drop as small as $240 \mathrm{~Pa}$ over the entire studied area that could be related to changes in the production rates at the underlying reservoir. These results thus provide a framework for monitoring the shallow crust with unprecedented accuracy with applications to active faults or volcano studies.

The seismic dataset was provided by Petroleum Development Oman (PDO) and is proprietary. It cannot be released to the public.

We acknowledge PDO and the Game Changer program at Shell for supporting this study, as well as the Ministry of Energy and Minerals of the Sultanate of Oman. This work received funding from the European Research Council (ERC) under Grant Agreement Number 817803 (FaultScan). We are grateful to Michel Campillo and Olivier Coutant for useful discussions, and to the reviewers of this 
paper for their insights. We also thank Saif Azri for his help.

\section{References}

Ahmadinejad, A. \& Kivi, I. R., 2021. An experimental investigation on the poroelastic response of a water-saturated limestone to hydrostatic compression, Bulletin of Engineering Geology and the Environment, pp. 3817-3832.

Brenguier, F., Campillo, M., Hadziioannou, C., Shapiro, N., Nadeau, R., \& Larose, E., 2008a. Postseismic relaxation along the San Andreas fault at Parkfield from continuous seismological observations, Science, 321(5895), 14781481.

Brenguier, F., Shapiro, N. M., Campillo, M., Ferrazzini, V., Duputel, Z., Coutant, O., \& Nercessian, A., 2008b. Towards forecasting volcanic eruptions using seismic noise, Nature Geoscience, 1(2), 126-130.

Brenguier, F., Campillo, M., Takeda, T., Aoki, Y., Shapiro, N., Briand, X., Emoto, K., \& Miyake, H., 2014. Mapping pressurized volcanic fluids from induced crustal seismic velocity drops, Science, 345(6192), 80-82.

Clements, T. \& Denolle, M. A., 2018. Tracking Groundwater Levels Using the Ambient Seismic Field, Geophysical Research Letters, 45(13), 6459-6465.

Doetsch, J., Gischig, V. S., Villiger, L., Krietsch, H., Nejati, M., Amann, F., Jalali, M., Madonna, C., Maurer, H., Wiemer, S., et al., 2018. Subsurface fluid pressure and rock deformation monitoring using seismic velocity observations, Geophysical Research Letters, 45(19), 10-389.

Kato, A. \& Ben-Zion, Y., 2021. The generation of large earthquakes, Nature Reviews Earth \& Environment, 2(1), 26-39. 
Niu, F., Silver, P. G., Daley, T. M., Cheng, X., \& Majer, E. L., 2008. Preseismic velocity changes observed from active source monitoring at the Parkfield SAFOD drill site, Nature, 454(7201), 204-208.

Olivier, G., Brenguier, F., Campillo, M., Roux, P., Shapiro, N., \& Lynch, R., 2015. Investigation of coseismic and postseismic processes using in situ measurements of seismic velocity variations in an underground mine, Geophysical Research Letters, 42(21), 9261-9269.

Poupinet, G., Ellsworth, W. L., \& Frechet, J., 1984. Monitoring velocity variations in the crust using earthquake doublets: an application to the Calaveras fault, California ( USA)., Journal of Geophysical Research, 89(B7), 57195731.

Rice, J. R. \& Cleary, M. P., 1976. Some basic stress diffusion solutions for fluid-saturated elastic porous media with compressible constituents, Reviews of Geophysics, 14(2), 227.

Rivet, D., Brenguier, F., \& Cappa, F., 2015. Improved detection of preeruptive seismic velocity drops at the Piton de La Fournaise volcano, Geophysical Research Letters, 42(15), 6332-6339.

Roeloffs, E., 1996. Poroelastic Techniques in the Study of Earthquake-Related Hydrologic Phenomena, Advances in Geophysics, 38(C), 135-195.

Scuderi, M. M. \& Collettini, C., 2016. The role of fluid pressure in induced vs. triggered seismicity: Insights from rock deformation experiments on carbonates, Scientific Reports, 6(January), 1-9.

Sens-Schönfelder, C. \& Wegler, U., 2006. Passive image interferometry and seasonal variations of seismic velocities at merapi volcano, indonesia, Geophysical research letters, $\mathbf{3 3}(21)$. 
Sens-Schönfelder, C., Pomponi, E., \& Peltier, A., 2014. Dynamics of Piton de la Fournaise volcano observed by passive image interferometry with multiple references, Journal of Volcanology and Geothermal Research, 276, 32-45.

Silver, P. G., Daley, T. M., Niu, F., \& Majer, E. L., 2007. Active Source Monitoring of Cross-Well Seismic Travel Time for Stress-Induced Changes, Bulletin of the Seismological Society of America, 97(1B), 281-293.

Snieder, R., Duran, A., \& Obermann, A., 2019. Locating velocity changes in elastic media with coda wave interferometry, chap, 6, 188-217.

Tsuji, S., Yamaoka, K., Ikuta, R., Kunitomo, T., Watanabe, T., Yoshida, Y., \& Katsumata, A., 2018. Secular and coseismic changes in S-wave velocity detected using ACROSS in the Tokai region, Earth, Planets and Space, 70(1).

Van Der Kamp, G. \& Gale, J. E., 1983. Theory of earth tide and barometric effects in porous formations with compressible grains, Water Resources Research, $\mathbf{1 9}(2), 538-544$.

Wang, Q. Y., Brenguier, F., Campillo, M., Lecointre, A., Takeda, T., \& Aoki, Y., 2017. Seasonal Crustal Seismic Velocity Changes Throughout Japan, Journal of Geophysical Research: Solid Earth, 122(10), 7987-8002.

Yamamura, K., Sano, O., Utada, H., Takei, Y., Nakao, S., \& Fukao, Y., 2003. Long-term observation of in situ seismic velocity and attenuation, Journal of Geophysical Research: Solid Earth (1978-2012), 108(B6). 
Figure 1: Seismic Monitoring Well (SMW) locations: a) Top view of the site of the experiments. b) 3D view of the wells. c) Average intersensor distance and depth inside the wells

Figure 2: Averaged daily spectrograms for the deepest sensors of SMW3 (a) and SMW5 (b). Every daily spectrograms is stacked using 15min bins.

Figure 3: Stability analysis of the EE component of the cross-correlations between SMW3 and SMW4 (top sensors). a) Cross-correlations according to calendar time. Each row corresponds to a 15-minute cross-correlation, with colors representing amplitude. Arrows on the x-axis represent the theoretical arrival time of S-waves for this sensor pair. b) Background: Nighttime cross-correlation according to calendar time. We distinguish three distinct time periods marked by dashed horizontal lines, corresponding to variations in the cross-correlations. The stacks of the $\mathrm{CC}$ for each time period are represented in black, blue and red, respectively. These stacks will be the reference for each time period. c) Cross-correlation coefficient between each reference and 3-night stacked correlations.

Figure 4: Velocity variations measured between SMW4 and SMW5. Each thin line corresponds to a sensor pair. The thick lines are the average velocity variations between the wells. Each color corresponds to a different reference used for the velocity measurement.

Figure 5: a) Pressure variation measured at the surface on the experiment site. b) Red line: effective pressure inverted from atmospheric pressure variations for a diffusivity $\mathrm{c}=100 \mathrm{~m}^{2} / \mathrm{s}$ and a depth $\mathrm{z}=100 \mathrm{~m}$. Black lines: velocity 
417

421

variations averaged over each well. Grey area: average errors on velocity measurements.

Figure 6: Effective pressure model. The top and bottom panels illustrate the effects of short and long term atmospheric variations on the pore pressure, respectively.

Figure 7: Representation of effective pressure models (blue lines) computed for various combination of depths and diffusivities. The average velocity change over the five wells (orange lines) was added to each plot for comparison. The estimated value for the sensitivity of velocity changes to effective pressure changes $\mathrm{K}$ computed over the first month is annotated on the bottom right corner of each plot. 


\section{${ }_{428}$ Figures}

a)

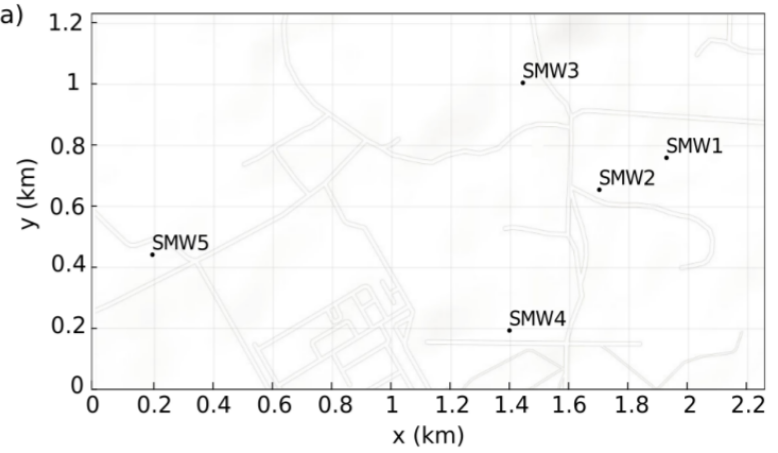

b) 0

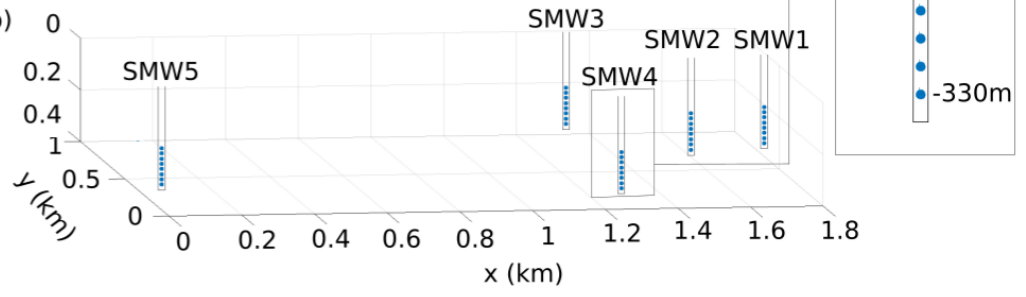

Figure 1: Seismic Monitoring Well (SMW) locations: a) Top view of the site of the experiments. b) 3D view of the wells. c) Average intersensor distances and depth inside the wells 

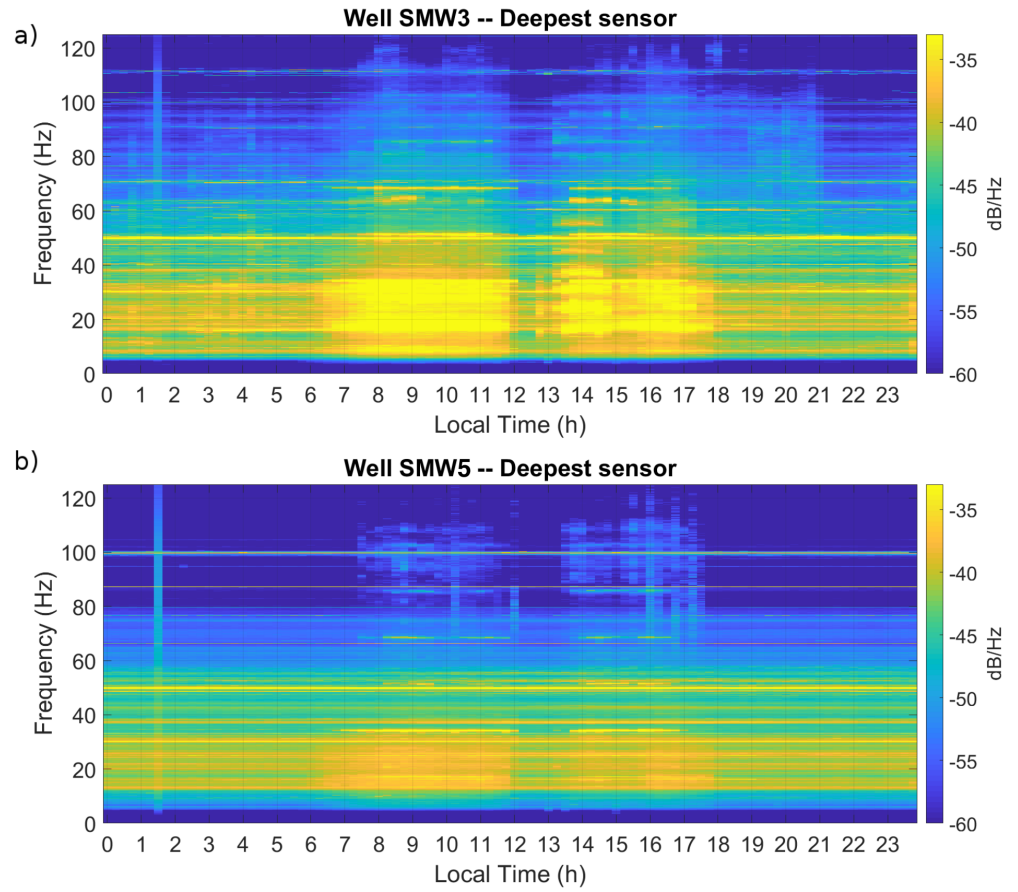

Figure 2: Averaged daily spectrograms for the deepest sensors of SMW3 (a) and SMW5 (b). Every daily spectrogram is stacked using 15min bins. 
a)
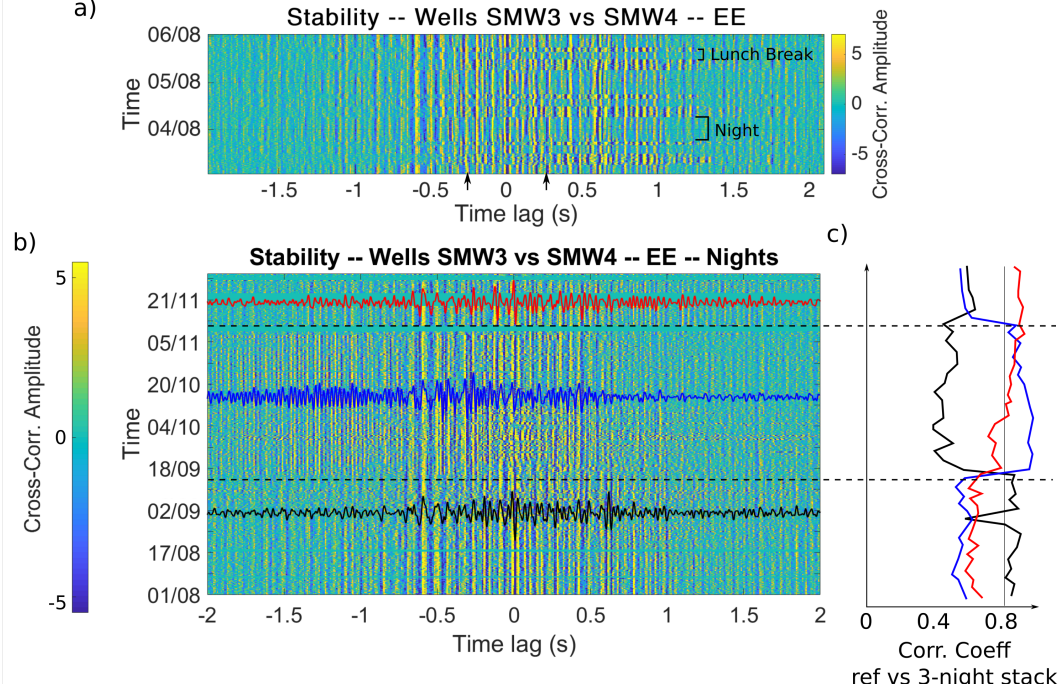

Figure 3: Stability analysis of the EE component of the cross-correlations between SMW3 and SMW4 (top sensors). a) Cross-correlations according to calendar time. Each row corresponds to a 15-minute cross-correlation, with colors representing amplitude. Arrows on the x-axis represent the theoretical arrival time of S-waves for this sensor pair b) Background: Nighttime cross-correlation according to calendar time. We distinguish three distinct time periods marked by dashed horizontal lines, corresponding to variations in the cross-correlations. The stacks of the CC for each time period are represented in black, blue and red, respectively. These stacks will be the reference for each time period. c) Cross-correlation coefficient between each reference and 3-night stacked correlations. 


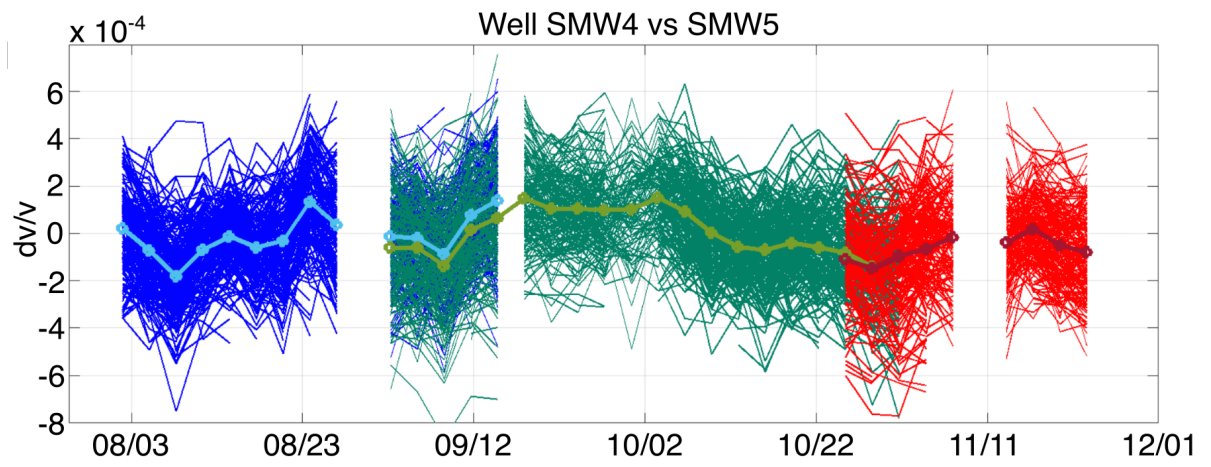

Figure 4: Velocity variations measured between SMW4 and SMW5. Each thin line corresponds to a sensor pair. The thick lines are the average velocity variations between the wells. Each color corresponds to a different reference used for the velocity measurement. 
a)

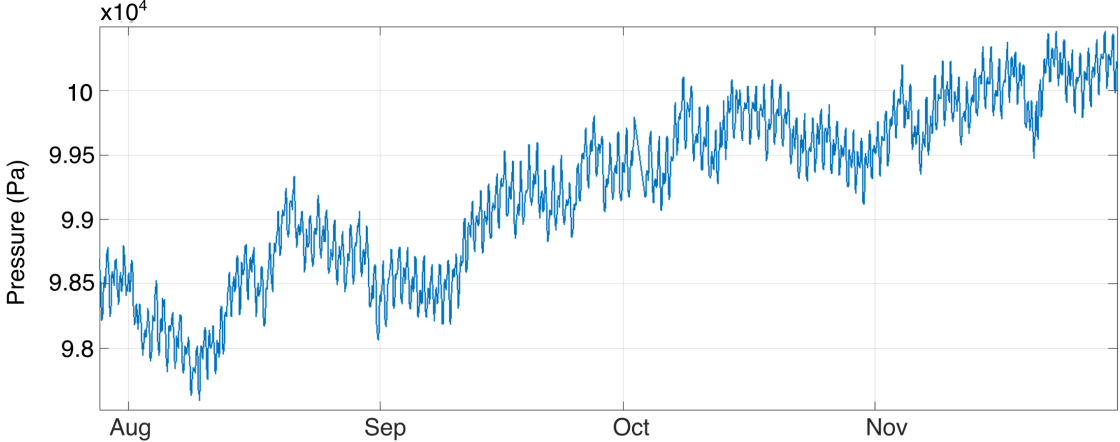

b)

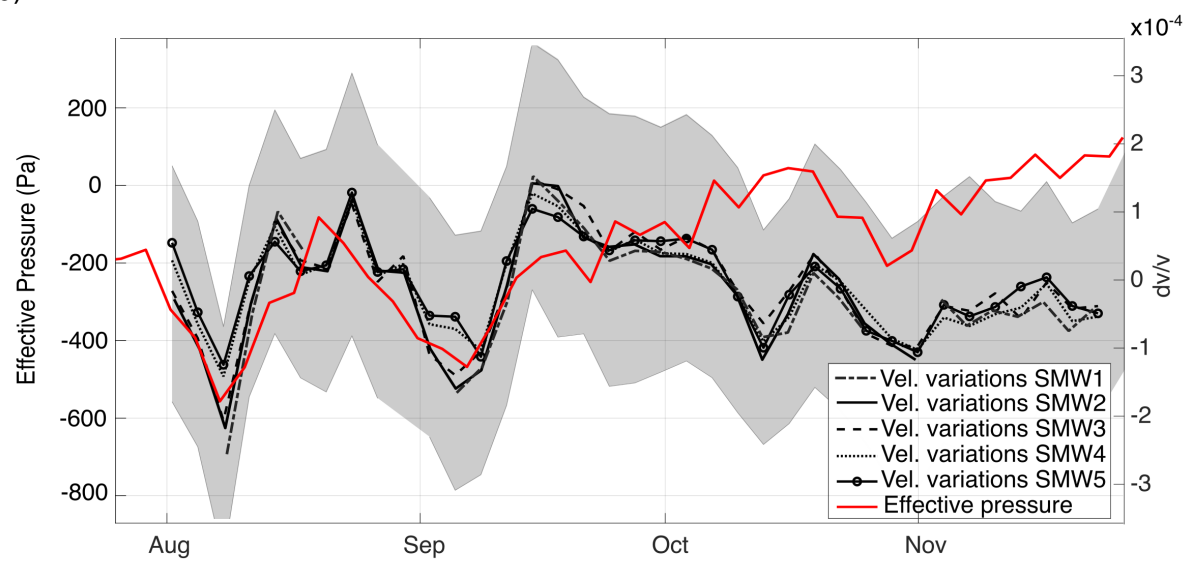

Figure 5: a) Pressure variation measured at the surface on the experiment site.

b) Red line: effective pressure inverted from atmospheric pressure variations for a diffusivity $\mathrm{c}=100 \mathrm{~m}^{2} / \mathrm{s}$ and a depth $\mathrm{z}=100 \mathrm{~m}$. Black lines: velocity variations averaged over each well. Grey area: average errors on velocity measurements. 
a) Short-term variations (weekly -- high $\omega$ )

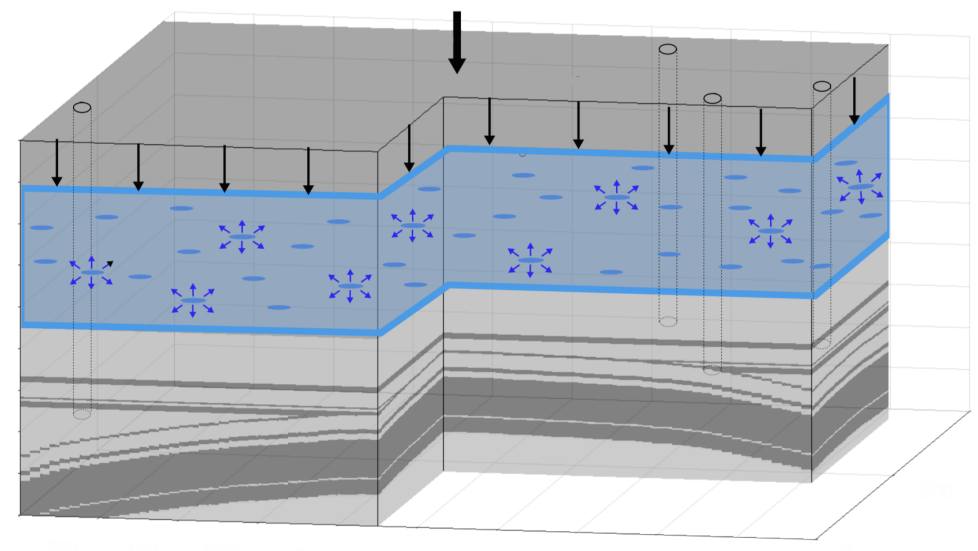

b) Long-term variations (yearly -- small $\omega$ )

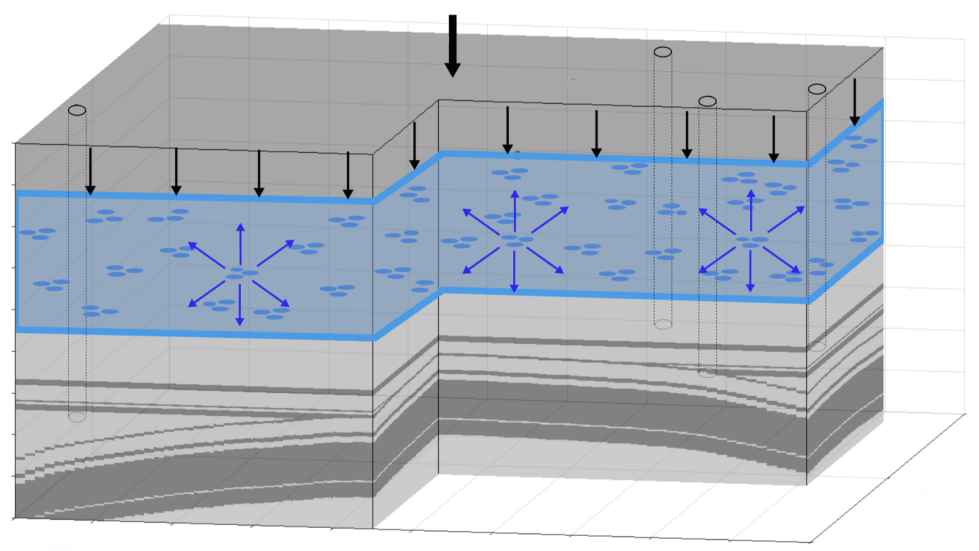
$\begin{array}{ll}\text { Wells } & \downarrow \text { Atmospheric pressure } \\ \text { Aquifer } & \quad>\text { Pore pressure effect }\end{array}$

Figure 6: Effective pressure model. Panels a and b illustrate the effects of short and long term atmospheric variations on the pore pressure, respectively. 

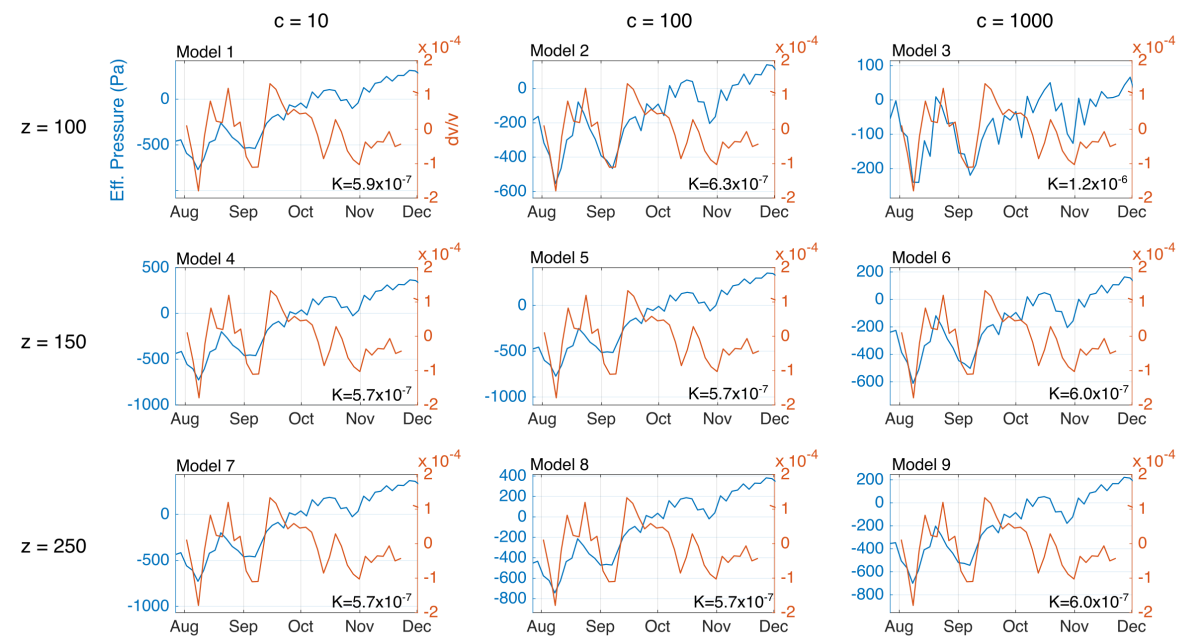

Figure 7: Representation of effective pressure models (blue lines) computed for various combination of depths and diffusivities. The average velocity change over the five wells (orange lines) was added to each plot for comparison. The estimated value for the sensitivity of velocity changes to effective pressure changes $\mathrm{K}$ computed over the first month is annotated on the bottom right corner of each plot. 